\title{
Potential Plasma Metabolic Biomarkers of Tourette Syndrome Discovery Based on Integrated Non- Targeted and Targeted Metabolomics Screening
}

\section{Leying Xi}

Nanjing University of Chinese Medicine

Fuqiong Zhou

Nanjing University of Chinese Medicine

Huan Sha

Nanjing University of Chinese Medicine

Weina Zhu

Nanjing University of Chinese Medicine

Xueling Hu

Nanjing University of Chinese Medicine

Jie Ruan

Nanjing University of Chinese Medicine

Yaruo Huang

Nanjing University of Chinese Medicine

Yajie Zhang ( $\square$ yajiezhang@vip.163.com )

Nanjing University of Chinese Medicine https://orcid.org/0000-0003-2510-6015

Hongyan Long

Nanjing University of Chinese Medicine

\section{Research}

Keywords: Non-targeted metabolomics, Targeted metabolomics, Tourette syndrome, Biomarkers, Metabolism pathway

Posted Date: December 16th, 2020

DOI: https://doi.org/10.21203/rs.3.rs-126790/v1

License: (1) (1) This work is licensed under a Creative Commons Attribution 4.0 International License.

Read Full License 


\section{Abstract}

Background Tourette syndrome (TS) is a chronic neuropsychiatric disorder, characterized by abnormal movements and phonations, tics, but accurate TS diagnosis remains challenging and indeed depends on its description of clinical symptoms. Our study was to identify some metabolites biomarkers based on non-targeted and targeted metabolomics.

Methods We conducted untargeted ultra-high-performance liquid chromatography -quadrupole time-off light mass spectrometry (UHPLC-Q-TOF/MS) for preliminary screening of potential biomarkers from 30 TS patients and 10 healthy controls. And then performed validation experiments based on targeted ultrahigh-performance liquid Chromatography-Triple Quadrupole-MS (UHPLC/MS/MS).

Results 1775 differentially metabolites were identified by partial least squares discriminant analysis (PLS-DA), fold change analysis, T-tests, and hierarchical clustering analysis (Adjusted $p$-value $<0.05$ and $|\log F C|>1)$. TS plasma samples were found differentiated from healthy samples in our approach. Further, the aspartate and asparagine metabolism pathway was considered as a significant enrichment pathway in TS progression based on the metabolite pathway enrichment analysis. For the 8 metabolites involved in this pathway that we detected, we then performed validation experiments based on targeted UHPLC/MS/MS from 35 TS patients and 14 healthy controls. T-test, Mann-Whitney U test, and receiver operating characteristic (ROC) curve analysis were used to determine potential biomarkers. Ultimately, LArginine and L-Pipecolic acid were validated as significantly differentiated metabolites $(p<0.05)$ with an AUC of $70 \%$ and $87 \%$.

Conclusions L-Arginine and L-Pipecolic acid were defined as potential biomarkers for TS diagnosis by the combined application of non-targeted and targeted metabolomics analysis.

\section{Key Messages}

- This is the first evaluation to discover plasma metabolic biomarkers of Tourette syndrome in the clinic.

- Non-targeted and targeted metabolomics is applied effectively for screening potential biomarkers in Tourette syndrome patients.

- L-Arginine and L-Pipecolic acid could be potential plasma metabolic biomarkers for Tourette syndrome diagnosis.

\section{Introduction}

Tourette syndrome (TS) is a childhood-onset chronic neuropsychiatric disorder characterized by chronic muscle movements and or vocal tics lasting more than one year[1]. Some children may have attentiondeficit/hyperactivity disorder (ADHD), anxiety, obsessive-compulsive disorder (OCD), and other comorbid behavioral syndromes[2], most children with TS can be expected to develop at least one comorbid 
disorder throughout their life, and more than half will develop two. Compared to tics, these comorbid conditions usually cause more impairments in patients with TS[3, 4]. Tics in TS typically start at 46 years old with motor movements like blinking, noise twitching, grimacing, and reach their worst severity around 10-12 years old $[5,6]$. Tics symptoms appear age dependently, showing a wax and wane course, and gradually ease by the late teens[5, 7].14 studies in mainstream school and school-age youngsters in the community reported prevalence figures for TS between the ages of 5 and 18 years very from 0.4$3.8 \%, 3989(0.949 \%)$ of the 420312 young people were diagnosed as having TS, therefore it was suggested that overall TS prevalence figure is $1 \%[8,9]$. In addition, studies have also shown that TS is more common in males than females, the ratio between male and female is about 3-4:1[4, 8]. Although the etiology and pathogenesis of TS remain uncertain, it has been proved to be closely related to genetic factors, neurobiochemical factors, environmental factors, psychology, and other factors[10-13]. Besides, Evidence has shown that the Cortico Striato Thalamo Cortex (CSTC) loop is related to the pathophysiology of TS closely[14].

To date, accurate TS diagnosis remains challenging and indeed depends on its clinical description of symptoms. The discovery of potential biomarkers that could help improve diagnosis is in high need. Usually, biomarkers are endogenous compounds and reflect the underlying disease characteristics. Metabolomics can detect, identify, and quantify small molecular endogenous metabolites to describe the biomarkers or characterize disease in biological samples, it concludes untargeted global profiling and targeted quantification. To the best of our knowledge, no publications on the metabolomics analysis of TS patients have been reported. Therefore, the purpose of the study was to investigate the potential biomarkers in the plasma in TS patients based on non-targeted and targeted metabolomics analysis.

\section{Materials And Methods}

\section{Clinical Participants}

From March 2019 to December 2019, TS patients and healthy controls were recruited from Nanjing Hospital of Chinese Medicine, Nanjing Hospital of Chinese Medicine Affiliated to Nanjing University of Chinese Medicine. TS patients were diagnosed by the Diagnostic and statistical manual of mental disorders $\left(\right.$ DSM $\left.-5^{\circledR}\right)[15]$. Healthy controls without TS and other known infections were included. All participants provided written informed consent. Finally, the total 49 participants have been recruited: 30 TS samples and 10 healthy controls for the untargeted UHPLC-Q-TOF/MS metabolomics analysis, the average age of the TS patients was 7.9 years, and 21 males (70.0\%) and 9 females (30.0\%) were included, the mean age of the healthy controls was 9.2 years, and 6 males $(60.0 \%)$ and 4 females (40\%) were included (sex $p=0.559$, age $p=0.211$ ); Another 35 TS samples and 14 healthy controls(include 30 TS samples and 10 healthy controls in untargeted analysis) for targeted UHPLC/MS/MS analysis, the average age of the TS patients was 8.086 years, and 24 males $(69.57 \%)$ and 11 females $(31.43 \%)$ were included, the mean age of the healthy controls was 9.5 years, and 9 males $(64.29 \%)$ and 5 females (35.71\%) were included(sex $p=0.773$, age $p=0.064)$. There was no statistical significance in both age and gender in TS and healthy controls. 


\section{Chemicals and reagents}

HPLC grade methanol (Thermo, A456-4); HPLC grade acetonitrile (Merck, 1499230-935); ammonium acetate (Sigma, 70221); ammonium hydroxide (Fluka). Analytical standards including L-Glutamic acid hydrochloride, D-Ornithine monohydrochloride, L-Ornithine monohydrochloride, D-Homoproline, D-Proline, L-Arginine, L-Pipecolic acid, L(-)- Carnitine were obtained from Shanghai Aladdin Biochemical Technology Co., Ltd. (Shanghai, China). Millipore-Q Water Purification System (Millipore, Germany) provided ultra-high purity water. All other chemicals and reagents were obtained from Merck Sigma-Aldrich (KGaA, Darmstadt, Germany).

\section{Sample preparation}

Blood samples were collected from each participant, then the samples were centrifuged $15 \min (3000 \times$ rcf, $4^{\circ} \mathrm{C}$ ) within 1 hour of collection. Each aliquot $(1 \mathrm{~mL})$ of the plasma samples were stored at $-80^{\circ} \mathrm{C}$ until the UHPLC-Q-TOF/MS and UHPLC/MS/MS processing. For the untargeted analysis, the plasma samples were thawed at $4^{\circ} \mathrm{C} .400 \mu \mathrm{L}$ of methanol/acetonitrile $(1: 1, \mathrm{v} / \mathrm{v})$ were added to $100 \mu \mathrm{L}$ of plasma After 60 sec. of the vortex, the mixture was stored at $-20^{\circ} \mathrm{C}$ for 1 hour to remove protein, and then centrifuged at $14,000 \times$ rcf for $20 \mathrm{~min}$ at $4^{\circ} \mathrm{C}$. The supernatants were subjected to UHPLC-Q-TOF/MS. -Quality control(QC) samples: $10 \mu \mathrm{L}$ of each plasma samples were mixed and treated in the same way as plasma samples. The QC samples were inserted in every 8 samples to monitor the system stability of UHPLC-Q-TOF/MS. For the targeted analysis, each plasma sample was thawed at $4^{\circ} \mathrm{C}$ for $30 \mathrm{~min}$ and $200 \mu \mathrm{L}$ aliquots were mixed with $200 \mu \mathrm{L}$ of methanol. Then the tubes were vortexed for $60 \mathrm{sec}$ and then centrifuged for 15 $\min \left(13,000 \times \mathrm{rcf}, 4^{\circ} \mathrm{C}\right)$. Preparation of standard solution: each $1 \mathrm{mg}$ analytical standard was dissolved in methanol $(1 \mathrm{mg} / \mathrm{ml})$ and then diluted in methanol $10 \mu \mathrm{g} / \mathrm{ml}$. The supernatant was transferred to an autosampler vial and a UHPLC/MS/MS method was used for the detection.

\section{UHPLC-Q-TOF/MS processing and data analysis}

UHPLC-Q-TOF/MS analysis was performed on an Agilent 1290 Infinity LC system (Agilent Technologies, Santa-Clara, California, USA), equipped with an AB SCIEX Triple TOF 5600 System (AB SCIEX, Framingham, MA, USA) in both positive and negative modes. Chromatographic separation was performed on ACQUITY HSS T3 $1.8 \mu \mathrm{m}(2.1 \times 100 \mathrm{~mm})$ columns. The column temperature was kept at $25^{\circ} \mathrm{C}$ and the flow rate was $0.3 \mathrm{~mL} / \mathrm{min}$. The UHPLC system consisting of water with $25 \mathrm{mM}$ ammonium acetate with $25 \mathrm{mM}$ ammonia (solvent $A$ ) and acetonitrile (solvent $B$ ). The gradient profile used was optimized as follows: $0-1 \mathrm{~min}, 95 \% \mathrm{~B} ; 1-14 \mathrm{~min}$, linearly changed to $65 \% \mathrm{~B} ; 14-16 \mathrm{~min}$, linearly changed to $40 \% \mathrm{~B} ; 16-18 \mathrm{~min}, 40 \% \mathrm{~B} ; 18-18.1 \mathrm{~min}$, linearly increased to $95 \% \mathrm{~B} ; 18.1-23 \mathrm{~min}, 95 \% \mathrm{~B}$. The sample injection volume was $2 \mu \mathrm{L}$.

Electrospray ionization (ESI) source conditions were set as follows: ion source gas 1(gas 1) was 60 psi; ion source gas 2(gas 2) was 60 psi; curtain gas(CUR) was 30 psi; source temperature was set to $600{ }^{\circ} \mathrm{C}$; ion spray voltage floating(ISVF) was 5000V (+) and -5000V (-). Information-dependent acquisition (IDA) is a product ion scan mode based on artificial intelligence, which was used to detect and identify MS/MS 
spectra. The parameters were set as follows: declustering potential (DP) was set to $60 \mathrm{~V}(+)$, and $-60 \mathrm{~V}(-)$; collision energy was $35 \pm 15 \mathrm{eV}$; exclude isotopes within $4 \mathrm{Da}$, candidate ions to monitor per cycle: 6 .

The raw data was generated by the Proteo Wizard MS converter tool, and processed by XCMS online software (https://xcmsonline.scripps.edu/landing_page.php?pgcontent=mainPage), including the nonlinear alignment, automatic integration, and peak extraction. After being normalized and integrated, MetaboAnalystR (3.0.3)[16] was employed for the statistical analysis (including PLS-DA analysis, hierarchical cluster analysis, Fold Change Analysis, and T-tests) and bioinformatics (pathway enrichment analysis) (www.metaboanalyst.ca). Significance was analyzed using Adjusted p-value $<0.05$ and |logFC| $>1$. For pathway enrichment analysis, two enrichment algorithms integrated mummichog and GSEA were used, and $P<0.05$ was considered statistically significant.

\section{Selection of metabolites for targeted metabolomics}

For the selection of biomarkers, the affected metabolic pathway containing abundant affected metabolites was the principal criterion. The preliminary identification of these metabolites was conducted by matching with the self-constructed databases (the secondary spectral library of standard samples established in the same experimental system, about 2500 kinds). In addition, The similarity values for the accuracy of compound identification and the number of differentially metabolites detected in each test sample were also important to reference factors[17]. Then the selected metabolites were identified by standards and tandem mass spectrometry.

\section{Sample processing and targeted UHPLC/MS/MS analyses}

The targeted validation of metabolites was carried out on an Agilent 1290 Infinity LC system (Agilent Technologies, Santa-Clara, California, USA) equipped with the Agilent 6460 triple quadrupole mass spectrometer (Agilent Technologies). The electrospray ionization(ESI) source was set in positive ion modes. Chromatographic separation was implemented on Agilent ZORBAX Hilic Plus (Agilent, USA) (50 $\times$ $2.1 \mathrm{~mm}, 1.8 \mu \mathrm{m}$ ) at a column temperature of $30^{\circ} \mathrm{C}$, a flow rate of $0.3 \mathrm{~mL} / \mathrm{min}$. The mobile phase $A$ consisted of solvent $A$ (methanol), and solvent $B(0.1 \%$ formic acid in water). The gradient profile was performed as follows: 0-1 min,5\%B; 1-2min, linearly increased to $90 \% \mathrm{~B} ; 2-3 \mathrm{~min}, 90 \% \mathrm{~B} ; 3-3.1 \mathrm{~min}$, returned to $5 \% ; 3.1-4 \mathrm{~min}, 5 \% \mathrm{~B}$. The sample injection volume was $5 \mu \mathrm{L}$. The optimized MS settings were: capillary voltage, $4000 \mathrm{v}(+), 3500 \mathrm{v}(-)$; the dry gas flow, $10 \mathrm{~L} \cdot \mathrm{min} \sim(-1)$, and the drying gas temperature, $350^{\circ} \mathrm{C}$. The quantitation was performed using multiple reaction monitoring (MRM) mode. The concentrations data of individual metabolites were calculated directly by UHPLC-MS/MS analysis $(\mathrm{nmol} / \mathrm{g})$.

In the UHPLC/MS/MS targeted analyses, the Student's t-test and the Mann-Whitney U test was used for comparisons between healthy and TS patients. All statistical analyses were analyzed by GraphPad Prism8 (GraphPad Software corporation, California, USA). Statistically, significance was defined by $\mathrm{P}<0.05$ (two-tailed). And the area under the ROC curves (AUC) was calculated to further analyze the differential metabolites. 


\section{Results}

\section{Non-targeted metabolomics analysis of plasma samples}

In the non-targeted metabolic analysis of 40 plasma samples, 9003 positive-mode features and 8790 negative-mode features were extracted from each sample. Before the subsequent analysis, we applied the quantile normalization and log transformation to the data, and the metabolomics data presented an average distribution after these processes (Fig. 1A, B). To identify the differences of metabolite profiles between TS patients and healthy controls, the PLS-DA model was performed in both positive and negative modes, including QC samples (Fig. 1C, D). There were significantly distinguished clusters between the TS patients and healthy controls, these results demonstrated that the metabolic profile was altered in the plasma of TS and healthy controls.

\section{Identification Of Differential Plasma Metabolites And Pathway Analysis}

The differentiated metabolites between the TS patients and healthy controls were further extracted by univariate statistical significance criteria (Adjusted p-value $<0.05$ and $|\log F C|>1$ ). The 1324 positivemode and 451 negative-mode different metabolite components were identified in the two groups. The Volcano Plot for the differential metabolites between the groups was presented in Fig. 2A and Fig. 2B. Based on the different metabolite components, the heat map of hierarchical clustering analysis was generated (Fig. 2C,D). The satisfactory discriminatory power could be found between the groups in the heatmap. Two enrichment algorithms contained mummichog and GSEA were used to analyze the enrichment pathways of differential metabolites (Fig. 3A[B). The pathways which had the most significant combined $p$-value were selected for graphic visual analysis in Fig. $3 \mathrm{C}$ (positive ion mode) and Fig. 3D (negative ion mode). There were 29 compounds matched in Aspartate and asparagine metabolism pathway and 42 compounds matched in Ascorbate (Vitamin C) and Aldarate Metabolism pathway. However, compared to healthy controls $₫$ the difference change in TS among these compounds performed a more consistent trend in Aspartate and asparagine metabolism pathway than Ascorbate (Vitamin C) and Aldarate Metabolism pathway. So we selected the 29 compounds matched in Aspartate and asparagine metabolism pathway for further study. Following matched the 29 compounds involved in the aspartate and asparagine metabolism pathway with the self-constructed database which has MS secondary spectrum data, we got 8 highly feasible compounds: L-Glutamate, Ornithine, D-Proline, LArginine, L-Pipecolic acid, L- Carnitine, D-Pipecolinic acid, and N-(omega)-Hydroxyarginine. These 8 metabolites were selected for subsequent targeted validation experiments. The basic characteristics of 8 metabolites were summarized in Table 1. 
Table 1

Differential metabolites identified by logFC and adjust. $p$ value

\begin{tabular}{|lllll|}
\hline Metabolites & M/Z & Retention Time & logFC & Adjust. $P$ Value \\
\hline L-Carnitine & 162.112 & 710.475 & 1.9373 & $<0.0001$ \\
\hline L-Glutamate & 148.0598 & 786.404 & 1.3103 & $<0.0001$ \\
\hline Ornithine & 133.0965 & 1009.645 & 1.1201 & 0.0011 \\
\hline D-Proline & 116.0699 & 1009.77 & 1.1579 & 0.0030 \\
\hline L-Arginine & 175.1186 & 1029.94 & 0.7928 & 0.0477 \\
\hline N-(omega)- Hydroxyarginine & 232.14 & 866.86 & 1.8824 & 0.0017 \\
\hline D-Pipecolinic acid & 130.0856 & 1047.88 & 1.0188 & 0.0004 \\
\hline L-Pipecolic acid & 147.1122 & 1047.48 & 1.2865 & $<0.0001$ \\
\hline
\end{tabular}

\section{Validated Ts Biomarkers Using A Targeted Metabolomics Analysis}

The specific concentration of 8 metabolites was determined by UHPLC/MS/MS (Supplementary Figure S2). Selected MRM transitions and the optimized conditions for MS were summarized in Table 2. Every metabolite linear standard curve was generated from the mixed standard solutions (Supplementary Figure S3). The $\mathrm{R}^{2}$ values of 8 metabolites stand curves linearity were all greater than 0.99 , these results indicate their accurate concentration calculation based on these curves (Supplementary Table S1). The concentration of L-Glutamate, D-Ornithine monohydrochloride, L-Arginine, L-Carnitine, D- Homoproline were increased in the TS plasmas than in the healthy plasmas, and the concentrations of D-Proline, LPipecolic acid, L-Ornithine monohydrochloride were decreased in the TS plasmas. 2 (L-Arginine, LPipecolic acid) of 8 verified metabolites had significant differences $(p<0.05)$ (Fig. 4). 
Table 2

Selected MS/MS channels and parameters

\begin{tabular}{|lllll|}
\hline component & parent ion & daughter ion & fragmentor/V & collis on energy/V \\
\hline L(-)-Carnitine & 162.0 & 60.0 & 115 & 17 \\
\hline L-Glutamic acid hydrochloride & 148.0 & 84.0 & 85 & 13 \\
\hline L-Ornithine monohydrochloride & 133.0 & 70.1 & 70 & 17 \\
\hline D-Proline & 116.0 & 70.1 & 85 & 21 \\
\hline L-Arginine & 175.1 & 70.2 & 110 & 29 \\
\hline D-Ornithine monohydrochloride & 133.0 & 70.0 & 60 & 21 \\
\hline D-Homoproline & 130.0 & 84.1 & 75 & 13 \\
\hline L-Pipecolicacid & 130.0 & 83.9 & 85 & 17 \\
\hline
\end{tabular}

\section{L-Arginine, L-Pipecolic acid could be as new biomarkers for TS diagnosis}

To evaluate the diagnostic value of 8 metabolites for TS, the ROC curve of them was performed and we calculated their area under the curve (AUC) and Youden indexes. As shown in Fig. 5, D-Ornithine (AUC = 56.9\%), D-Proline (AUC = 35.5\%), D-Homoproline (AUC = 41.1\%), L-Glutamate (AUC = 41.1\%), L-Glutamate $(A \cup C=62.2 \%)$, L-Arginine (AUC = 70\%), L-Ornithine (AUC = 47.6\%), D-Pipecolinic acid (AUC = 87.1\%), LCarnitine (AUC = 51.2\%). L-Arginine and L-Pipecolic acid had a better diagnostic value for TS (AUC > 70\%). The optimal threshold was $99 \mathrm{ng} / \mu \mathrm{l}$ for L-Glutamate and $21 \mathrm{ng} / \mu \mathrm{l}$ for D-Pipecolinic acid in the test plasma.

\section{Discussion}

Tourette Syndrome is a complex neurological disorder characterized by repetitive, sudden, involuntary motor, and phonic tics. Most children with TS can be estimated to develop other associated comorbid conditions. However, there are problems with the actual diagnosis of TS, the diagnostic concept is subjective and indeed depends on its clinical description of symptoms. There is a high need to discover some biomarkers that could help improve diagnosis. Metabolomics provides us a unique perspective to understand the regulation of metabolic networks in the biological system. Furthermore, it is also emerging as a new tool to research the central nervous system, and there are already some researches about metabolomic signatures in Schizophrenia, depression, bipolar disease[18-21]. As our best knowledge, this was the first study to explore potential plasma metabolic biomarkers of TS through nontargeted combined with targeted metabolic profiling. 
In the untargeted metabolomics analysis, there was a clear distinction between the TS plasma and healthy plasma, and this also reflected our strict inclusion criteria of TS patients based on existing clinical diagnosis. Furthermore, aspartate and asparagine metabolism pathway were found to be significantly affected by TS, and 8 differential metabolites in this pathway were identified by matching with the self-constructed databases (D-Ornithine, D-Proline, D-Homoproline, L-Glutamate, L-Glutamate, LArginine, L-Ornithine, D-Pipecolinic acid, L-Carnitine). To further validate the potential biomarkers in TS patients, UHPLC/MS/MS targeted quantitative analysis was performed. At this stage, we collected TS patients as possible as we could. However, the sample size of TS patients in the study is limited because of the low incidence and informed consent of parents of children. The final results showed that only 2 (LArginine, L-Pipecolic acid) of 8 metabolites had statistical differences between TS patients and healthy controls $(p<0.05)$. Coincidentally, L-Arginine and L-Pipecolic acid were also performed in the better area under the curve among 8 metabolites. Limited by the sample size, limited by the sample size, we realized this study should be collected more clinical samples to construct more validation cohort for more precise diagnosis.

The etiology and pathogenesis of TS are still uncertain, but the Cortico Striato Thalamo Cortex (CSTC) circuit appears closely related to the pathophysiology of TS, and some amino acids including GABA and glutamate act as the neurotransmitters and neuromodulator, play a key role in CSTC circuitry and participate in habitual behavior formation and the pathophysiology of tics[22-24]. As the main regulatory element in the CSTC circuit, large studies also reported the role of the striatum in TS[25-27]. Studies found glutamate was increased in TS, while in our study glutamate showed no statistical differences between TS patients and healthy controls, it may be related to our limited size of samples. Besides, the aspartate and asparagine metabolism pathway, L-Arginine, L-Pipecolic acid we found in the study also indicate that amino acids participant the pathogenesis of TS. As precursors and intermediates of neurotransmitters, amino acids play an important role in neurotrophic, development, and information transmission. It is difficult to determine the amino acids level of brain tissue and cerebrospinal fluid in TS patients, while the free amino acids in human body fluid could be stable in TS active period. In addition, amino acids could pass through the blood-brain barrier, so the level of amino acids in the blood can also indirectly reflect the situation of amino acids in the brain.

Aspartate and asparagine are two non-essential amino acids with similar structures. Aspartate exists in two enantiomeric forms, L-aspartic acid, and D-aspartic acid. As excitatory neurotransmitters in the central nervous system, aspartate takes part in the long-range information exchange via activation of glutamate receptor channels[28]. While high levels of aspartate could reduce synaptic plasticity, impair cognitive function, and early spatial memory[29, 30]. N-Methyl-D-aspartic acid (NMDA) is a high-energy form of aspartic acid and one of a well-known agonist for a class of glutamate receptors. Aspartate can selectively activate extrasynaptic NR1-NR2B NMDA receptors. Aspartate may participate in immature neurons through activates the NR1-NR2B receptors, results in the substantial $\mathrm{Ca}^{2+}$ influx, then activates cAMP-dependent gene transcription; and inhibits cAMP-response element-binding protein (CREB) function, reduce the expression of brain-derived neurotrophic factor (BDNF), and induce excitotoxic 
neuronal death in mature neurons[31]. Excessive activation of NMDA receptors is associated with memory, learning impairment, and excitotoxic cell death[32-35]. It is interesting to note that previous studies showed the number of NMDA receptor in the hippocampus is decreased by aging[34], while symptom intensity and frequency of TS also decreases with age, whether this feature of TS is related to NMDA receptor still needs to be further studied. In addition, $N$-acetylaspartate (NAA), a non-invasive marker for neuronal health, which is synthesized from aspartate and acetyl-coenzyme A in neurons, reflects the extent of neuronal impairment and dysfunction. Several studies have found that the decreased concentration of NAA is involved in neuropsychiatric disorders listed in DSM-IV 1R, the acknowledged compendium of clinical psychiatric disease[36, 37]. There are a few kinds of research between NAA and TS. One study found lower levels of $\mathrm{N}$-acetylaspartate in the left putamen and the frontal cortex in TS plasma and suggested the compromised neuronal integrity and insufficient density of neuronal and nonneuronal cells[38]. Taken together, these researches suggested that aspartate and asparagine metabolism pathway has a close relationship with the pathogenesis of TS.

In the study, the levels of L-arginine were up-regulated in TS patients. L-arginine is a semi-essential amino acid involves in the synthesis of L-ornithine, L-glutamate and polyamines, and a precursor for nitric oxide (NO) synthesis[39]. The L-Arginine/NO pathway is involved in physiological processes such as vasodilation, memory, neuroprotection, and immune defense in systems of the cardiovascular, immune, and nervous systems[40,41]. The effects of L-Arginine in the nervous system are often attributed to NO. In the nervous system, NO acts as a neurotransmitter, and play an important role in synaptic plastic, neural development, regeneration, transcriptional activity, learning and memory, and neuroprotection[4244]. Small quantities of NO are neuroprotective, excessive NO becomes noxious which could cause cell damage and involved in various disorders like major depressive disorder (MDD), autism spectrum disorder (ASD), obsessive-compulsive disorder (OCD), Alzheimer's disease (AD), attention deficit hyperactivity disorder(ADHD) and other neurodegenerative disorders[40, 45-49]. The role of L-Arginine and NO in Tourette syndrome has not been explored, our results first time showed the concentration of LArginine was higher in the TS patients, whether the L-Arginine/NO pathway is involved in the development of TS needs to be further studied.

Pipecolic acid (PA) is a metabolite of lysine and has two different enantiomers, including L-Pipecolic acid (L-PA) and D-Pipecolic acid (D-Homoproline; D-PA). L-PA is a significant marker for the diagnosis of peroxisomal disorders. D-PA is believed to originate mainly derived from the catabolism of dietary lysine by intestinal bacteria and was found to increase in patients with liver diseases[50,51]. PA is known to be a GABA receptor agonist, it could inhibit the neuronal GABA uptake and/or enhance its release, while D-PA was found more effective in restraining the actions of the central GABA system than L-PA acid [52, 53]. GABA is a major inhibitory neurotransmitter involved in the CSTC pathway, its dysfunction has a close relationship with TS. In the study, D-Pipecolic acid increased in TS and displayed moderate efficiency $(A \cup C=87.15)$ as a potential biomarker. We speculated that D-PA may participate in the pathophysiology of TS through the central GABA system. 
However, there are two limitations to the study. First, 49 TS patients and healthy controls were recruited, however, the size of samples was limited. Second, the global generalizability of the findings may be affected, cause all participants recruited in this study were from China. Further, a large number of participants are needed to validate the potential utility of these plasma metabolic biomarkers in the diagnosis of TS.

\section{Conclusion}

In summary, we employed a UHPLC/Q-TOF/MS non-targeted analysis and UHPLC/MS/MS targeted quantitative analysis to identify plasma metabolic profile in TS. Aspartate and asparagine metabolism pathway was found to be significantly affected by TS. L-Arginine, L-Pipecolic acid may be used as potential diagnostic biomarkers of TS. Furthermore, our study also confirmed that the imbalance of amino acid neurotransmitters is closely associated with the pathophysiology of TS, while the role of amino acid in TS still deserves further exploration.

\section{Declarations}

\section{Ethics approval and consent to participate}

The human study was conducted under protocols approved by the Ethical Committee of the Nanjing Hospital of Chinese Medicine. Written informed consents were obtained from all study subjects before participation.

\section{Consent for publication}

Not Applicable

\section{Availability of data and material}

The data that support the findings of this study are available from the corresponding author upon reasonable request.

\section{Code availability}

Raw metabolomics data were processed using ProteoWizard and XCMS software. Metabolomics analyses were performed using R package MetaboAnalystR (3.0.3). Statistical analyses were performed using $R$ version 4.0.3 software.

\section{Competing interests}

The authors declare that they have no competing interests.

\section{Funding}


This work was supported by the National Nature Science Foundation of China (81774364) and the Zhang Biao Famous T.C.M. Doctor Studio of Nanjing Health Commission (ZB-2017-NJ).

\section{Author contribution statement}

Conceptualization, Yajie Zhang and Hongyan Long; Data curation, Fuqiong Zhou and Yajie Zhang; Formal analysis, Yajie Zhang; Funding acquisition, Hongyan Long; Investigation, Leying Xi, Fuqiong Zhou and Weina Zhu; Methodology, Leying Xi, Huan Sha, Weina Zhu and Yajie Zhang; Project administration, Hongyan Long; Resources, Weina Zhu, Xueling Hu and Hongyan Long; Software, Fuqiong Zhou and Yaruo Huang; Supervision, Hongyan Long; Validation, Leying Xi, Fuqiong Zhou, Huan Sha, Weina Zhu and Jie Ruan; Visualization, Yajie Zhang; Writing - original draft, Leying Xi; Writing - review \& editing, Yajie Zhang and Hongyan Long.

\section{Acknowledgements}

We thank the department of Central Laboratory in Nanjing Hospital of Chinese Medicine and Chunhua Ma, Mengjiao Zhang, and Chunyan Wang for their help in our laboratory.

\section{References}

1. Leckman JF. Tourette's syndrome. Lancet. 2002;360:1577-86. DOI 10.1016/S0140-6736(02)115261.

2. Cravedi E, Deniau E, Giannitelli M, Pellerin H, Czernecki V, Priou T, Xavier J, Consoli A, Hartmann A, Cohen D. Disentangling Tourette syndrome heterogeneity through hierarchical ascendant clustering. Dev Med Child Neurol. 2018;60:942-50. DOI 10.1111/dmcn.13913.

3. Bernard BA, Stebbins GT, Siegel S, Schultz TM, Hays C, Morrissey MJ, Leurgans S, Goetz CG. Determinants of quality of life in children with Gilles de la Tourette syndrome. Movement disorders: official journal of the Movement Disorder Society. 2009;24:1070-3. DOI 10.1002/mds.22487.

4. Hirschtritt ME, Lee PC, Pauls DL, Dion Y, Grados MA, Illmann C, King RA, Sandor P, McMahon WM, Lyon GJ, et al. Lifetime prevalence, age of risk, and genetic relationships of comorbid psychiatric disorders in Tourette syndrome. JAMA Psychiatry. 2015;72:325-33. DOI 10.1001/jamapsychiatry.2014.2650.

5. Bloch MH, Leckman JF. Clinical course of Tourette syndrome. J Psychosom Res. 2009;67:497-501. DOI 10.1016/j.jpsychores.2009.09.002.

6. Leckman JF, Zhang H, Vitale A, Lahnin F, Lynch K, Bondi C, Kim YS, Peterson BS. Course of tic severity in Tourette syndrome: the first two decades. Pediatrics. 1998;102:14-9. DOI 10.1542/peds.102.1.14.

7. Peterson BS, Leckman JF. The temporal dynamics of tics in Gilles de la Tourette syndrome. Biol Psychiatry. 1998;44:1337-48. DOI 10.1016/s0006-3223(98)00176-0. 
8. Robertson MM. The prevalence and epidemiology of Gilles de la Tourette syndrome. Part 1: the epidemiological and prevalence studies. J Psychosom Res. 2008;65:461-72. DOI 10.1016/j.jpsychores.2008.03.006.

9. Robertson MM. The prevalence and epidemiology of Gilles de la Tourette syndrome. Part 2: tentative explanations for differing prevalence figures in GTS, including the possible effects of psychopathology, aetiology, cultural differences, and differing phenotypes. J Psychosom Res. 2008;65:473-86. DOI 10.1016/j.jpsychores.2008.03.007.

10. Brander G, Rydell M, Kuja-Halkola R, Fernandez de la Cruz L, Lichtenstein P, Serlachius E, Ruck C, Almqvist C, D'Onofrio BM, Larsson $\mathrm{H}$, et al. Perinatal risk factors in Tourette's and chronic tic disorders: a total population sibling comparison study. Mol Psychiatry. 2018;23:1189-97. DOI 10.1038/mp.2017.31.

11. Gagne JP. The psychology of Tourette disorder: Revisiting the past and moving toward a cognitivelyoriented future. Clin Psychol Rev. 2019;67:11-21. DOI 10.1016/j.cpr.2018.09.005.

12. Hongyan L, Mengjiao Z, Chunyan W, Yaruo H. Rhynchophyllin attenuates neuroinflammation in Tourette syndrome rats via JAK2/STAT3 and NF-kappaB pathways. Environ Toxicol. 2019;34:111420. DOI 10.1002/tox.22813.

13. Qi Y, Zheng Y, Li Z, Liu Z, Xiong L. Genetic Studies of Tic Disorders and Tourette Syndrome. Methods in molecular biology. 2019;2011:547-71. DOI 10.1007/978-1-4939-9554-7\$432.

14. Albin RL, Mink JW. Recent advances in Tourette syndrome research. Trends Neurosci. 2006;29:17582. DOI 10.1016/j.tins.2006.01.001.

15. Association AP. (2013) Diagnostic and statistical manual of mental disorders (DSM-5®)American Psychiatric Pub.

16. Pang Z, Chong J, Li S, Xia J. (2020) MetaboAnalystR 3.0: Toward an Optimized Workflow for Global Metabolomics. Metabolites 10. DOI 10.3390/metabo10050186.

17. Chen Y, Ma Z, Shen X, Li L, Zhong J, Min LS, Xu L, Li H, Zhang J, Dai L. (2018) Serum Lipidomics Profiling to Identify Biomarkers for Non-Small Cell Lung Cancer. Biomed Res Int 2018: 5276240. DOI 10.1155/2018/5276240.

18. Kaddurah-Daouk R, Krishnan KR. Metabolomics: a global biochemical approach to the study of central nervous system diseases. Neuropsychopharmacology. 2009;34:173-86. DOI 10.1038/npp.2008.174.

19. Kaddurah-Daouk R, McEvoy J, Baillie RA, Lee D, Yao JK, Doraiswamy PM, Krishnan KR. Metabolomic mapping of atypical antipsychotic effects in schizophrenia. Mol Psychiatry. 2007;12:934-45. DOI 10.1038/sj.mp.4002000.

20. Lan MJ, McLoughlin GA, Griffin JL, Tsang TM, Huang JT, Yuan P, Manji H, Holmes E, Bahn S. Metabonomic analysis identifies molecular changes associated with the pathophysiology and drug treatment of bipolar disorder. Mol Psychiatry. 2009;14:269-79. DOI 10.1038/sj.mp.4002130.

21. Paige LA, Mitchell MW, Krishnan KR, Kaddurah-Daouk R, Steffens DC. A preliminary metabolomic analysis of older adults with and without depression. Int J Geriatr Psychiatry. 2007;22:418-23. DOI 
10.1002/gps.1690.

22. Gasbarri A, Pompili A, Packard MG, Tomaz C. Habit learning and memory in mammals: behavioral and neural characteristics. Neurobiol Learn Mem. 2014;114:198-208. DOI 10.1016/j.nlm.2014.06.010.

23. Mahone EM, Puts NA, Edden RAE, Ryan M, Singer HS. GABA and glutamate in children with Tourette syndrome: A (1)H MR spectroscopy study at 7T. Psychiatry Res Neuroimaging. 2018;273:46-53. DOI 10.1016/j.pscychresns.2017.12.005.

24. Singer HS. Habitual and goal-directed behaviours and Tourette syndrome. Brain. 2016;139:312-6. DOI 10.1093/brain/awv378.

25. Caligiore D, Mannella F, Arbib MA, Baldassarre G. Dysfunctions of the basal ganglia-cerebellarthalamo-cortical system produce motor tics in Tourette syndrome. PLoS Comput Biol. 2017;13:e1005395. DOI 10.1371/journal.pcbi.1005395.

26. Kataoka Y, Kalanithi PS, Grantz H, Schwartz ML, Saper C, Leckman JF, Vaccarino FM. Decreased number of parvalbumin and cholinergic interneurons in the striatum of individuals with Tourette syndrome. J Comp Neurol. 2010;518:277-91. DOI 10.1002/cne.22206.

27. Rizzo F, Nespoli E, Abaei A, Bar-Gad I, Deelchand DK, Fegert J, Rasche V, Hengerer B, Boeckers TM. Aripiprazole Selectively Reduces Motor Tics in a Young Animal Model for Tourette's Syndrome and Comorbid Attention Deficit and Hyperactivity Disorder. Front Neurol. 2018;9:59. DOI 10.3389/fneur.2018.00059.

28. Toyota M, Spencer D, Sawai-Toyota S, Jiaqi W, Zhang T, Koo AJ, Howe GA, Gilroy S. Glutamate triggers long-distance, calcium-based plant defense signaling. Science. 2018;361:1112-5. DOI 10.1126/science.aat7744.

29. Cui $B$, Wu M, She $X$, Liu H. Impulse noise exposure in rats causes cognitive deficits and changes in hippocampal neurotransmitter signaling and tau phosphorylation. Brain research. 2012;1427:35-43. DOI 10.1016/j.brainres.2011.08.035.

30. Humphries $P$, Pretorius $E$, Naude $H$. Direct and indirect cellular effects of aspartame on the brain. Eur J Clin Nutr. 2008;62:451-62. DOI 10.1038/sj.ejcn.1602866.

31. Nadler JV. Aspartate release and signalling in the hippocampus. Neurochem Res. 2011;36:668-76. DOI 10.1007/s11064-010-0291-3.

32. Gillessen T, Budd SL, Lipton SA. Excitatory amino acid neurotoxicity. Adv Exp Med Biol. 2002;513:340. DOI 10.1007/978-1-4615-0123-7\$41.

33. Tsoka P, Barbisan PR, Kataoka K, Chen XN, Tian B, Bouzika P, Miller JW, Paschalis El, Vavvas DG. NLRP3 inflammasome in NMDA-induced retinal excitotoxicity. Exp Eye Res. 2019;181:136-44. DOI 10.1016/j.exer.2019.01.018.

34. Yamada K, Nabeshima T (1998) Changes in NMDA receptor/nitric oxide signaling pathway in the brain with aging. Microsc Res Tech 43: 68-74. DOI 10.1002/(SICI)1097-0029(19981001)43:1 < 68::AID-JEMT10 > 3.0.C0;2-W 
35. Zhou JJ, Li DP, Chen SR, Luo Y, Pan HL. The alpha2delta-1-NMDA receptor coupling is essential for corticostriatal long-term potentiation and is involved in learning and memory. J Biol Chem. 2018;293:19354-64. DOI 10.1074/jbc.RA118.003977.

36. Moffett JR, Arun P, Ariyannur PS, Namboodiri AM. N-Acetylaspartate reductions in brain injury: impact on post-injury neuroenergetics, lipid synthesis, and protein acetylation. Front Neuroenergetics. 2013;5:11. DOI 10.3389/fnene.2013.00011.

37. Moffett JR, Ross B, Arun P, Madhavarao CN, Namboodiri AM. N-Acetylaspartate in the CNS: from neurodiagnostics to neurobiology. Prog Neurobiol. 2007;81:89-131. DOI 10.1016/j.pneurobio.2006.12.003.

38. DeVito TJ, Drost DJ, Pavlosky W, Neufeld RW, Rajakumar N, McKinlay BD, Williamson PC, Nicolson R. Brain magnetic resonance spectroscopy in Tourette's disorder. J Am Acad Child Adolesc Psychiatry. 2005;44:1301-8. DOI 10.1097/01.chi.0000181046.52078.f4.

39. Boger RH. The pharmacodynamics of L-arginine. Altern Ther Health Med. 2014;20:48-54.

40. Calabrese V, Mancuso C, Calvani M, Rizzarelli E, Butterfield DA, Stella AM. Nitric oxide in the central nervous system: neuroprotection versus neurotoxicity. Nat Rev Neurosci. 2007;8:766-75. DOI 10.1038/nrn2214.

41. Shinde UA, Mehta AA, Goyal RK. Nitric oxide: a molecule of the millennium. Indian J Exp Biol. 2000;38:201-10.

42. Bradley SA, Steinert JR. (2016) Nitric Oxide-Mediated Posttranslational Modifications: Impacts at the Synapse. Oxid Med Cell Longev 2016: 5681036. DOI 10.1155/2016/5681036.

43. Hardingham N, Dachtler J, Fox K. The role of nitric oxide in pre-synaptic plasticity and homeostasis. Front Cell Neurosci. 2013;7:190. DOI 10.3389/fncel.2013.00190.

44. Yun HY, Dawson VL, Dawson TM. Nitric oxide in health and disease of the nervous system. Mol Psychiatry. 1997;2:300-10. DOI 10.1038/sj.mp.4000272.

45. Avcil S, Uysal P, Yenisey C, Abas BI. (2019) Elevated Melatonin Levels in Children With Attention Deficit Hyperactivity Disorder: Relationship to Oxidative and Nitrosative Stress. J Atten Disord: 1087054719829816. DOI 10.1177/1087054719829816.

46. Gawali NB, Chowdhury AA, Kothavade PS, Bulani VD, Nagmoti DM, Juvekar AR. Involvement of nitric oxide in anticompulsive-like effect of agmatine on marble-burying behaviour in mice. Eur $\mathrm{J}$ Pharmacol. 2016;770:165-71. DOI 10.1016/j.ejphar.2015.11.044.

47. Ghasemi M. Nitric oxide: Antidepressant mechanisms and inflammation. Adv Pharmacol. 2019;86:121-52. DOI 10.1016/bs.apha.2019.04.004.

48. Knott AB, Bossy-Wetzel E. Nitric oxide in health and disease of the nervous system. Antioxid Redox Signal. 2009;11:541-54. DOI 10.1089/ARS.2008.2234.

49. Yui K, Kawasaki Y, Yamada H, Ogawa S. Oxidative Stress and Nitric Oxide in Autism Spectrum Disorder and Other Neuropsychiatric Disorders. CNS Neurol Disord Drug Targets. 2016;15:587-96. DOI 10.2174/1871527315666160413121751. 
50. Fujita T, Hada T, Higashino K. Origin of D- and L-pipecolic acid in human physiological fluids: a study of the catabolic mechanism to pipecolic acid using the lysine loading test. Clin Chim Acta. 1999;287:145-56. DOI 10.1016/s0009-8981(99)00129-1.

51. Rashed MS, Al-Ahaidib LY, Aboul-Enein HY, Al-Amoudi M, Jacob M. Determination of L-pipecolic acid in plasma using chiral liquid chromatography-electrospray tandem mass spectrometry. Clin Chem. 2001;47:2124-30.

52. Beitz AJ, Larson AA. Inhibition of intrathecally administered picrotoxin- and bicuculline-induced convulsions in mice by pipecolic acid or GABA. Eur J Pharmacol. 1985;114:181-7. DOI 10.1016/0014-2999(85)90626-0.

53. Gutierrez MC, Delgado-Coello BA. Influence of pipecolic acid on the release and uptake of [3H]GABA from brain slices of mouse cerebral cortex. Neurochem Res. 1989;14:405-8. DOI 10.1007/BF00964852.

\section{Supplementary Table}

Supplementary Table S1 The stand curve formulas of metabolite standards.

\begin{tabular}{|c|c|c|}
\hline component & stand curve & $\mathrm{R}^{2}$ \\
\hline L(-)-Carnitine & $y=4.3390 * x-69.8655$ & 0.9972 \\
\hline L-Glutamic acid hydrochloride & $y=0.3286^{*} x-1.9465$ & 0.9983 \\
\hline L-Ornithine monohydrochloride & $y=1.2999 * x-26.4997$ & 0.9970 \\
\hline D-Proline & $y=1.0752^{*} x+22.1328$ & 0.9998 \\
\hline L-Arginine & $y=6.0785^{\star} x+156.3409$ & 0.9982 \\
\hline D-Ornithine monohydrochloride & $y=1.1636^{*} x-22.2691$ & 0.9976 \\
\hline D-Homoproline & $y=5.1628^{*} x-95.0264$ & 0.9997 \\
\hline L-Pipecolicacid & $y=5.7487 * x-180.3461$ & 0.9975 \\
\hline
\end{tabular}

\section{Figures}


A
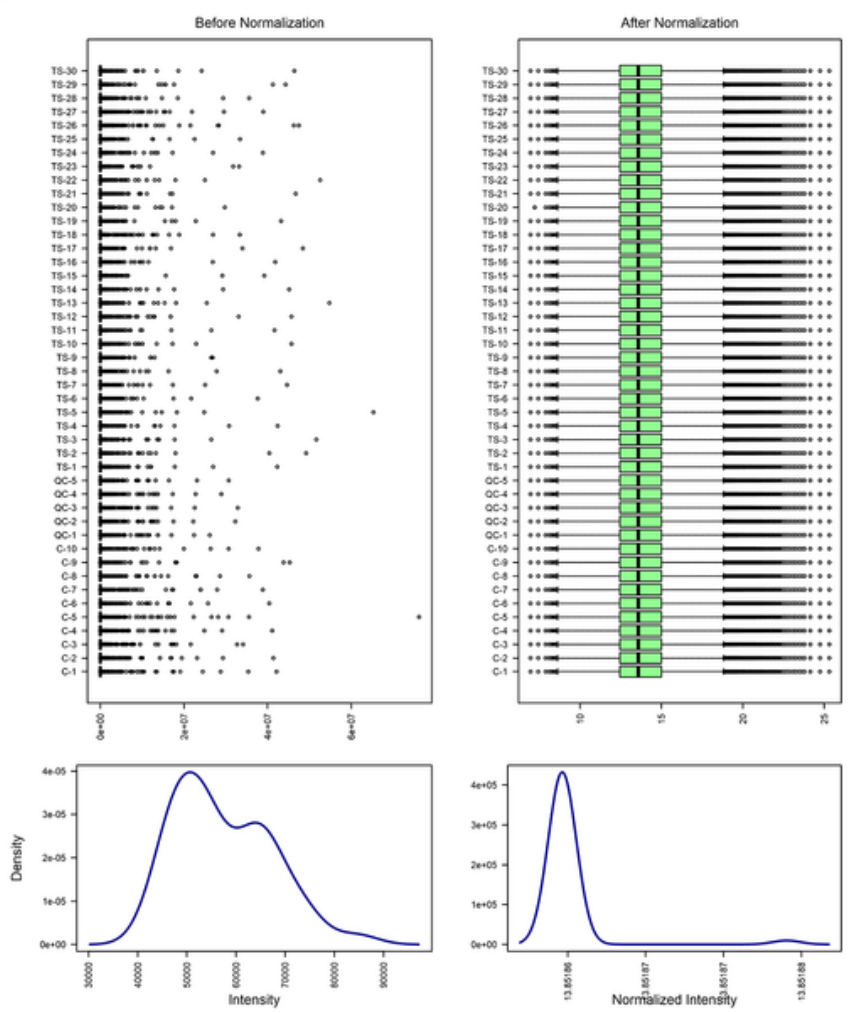

C

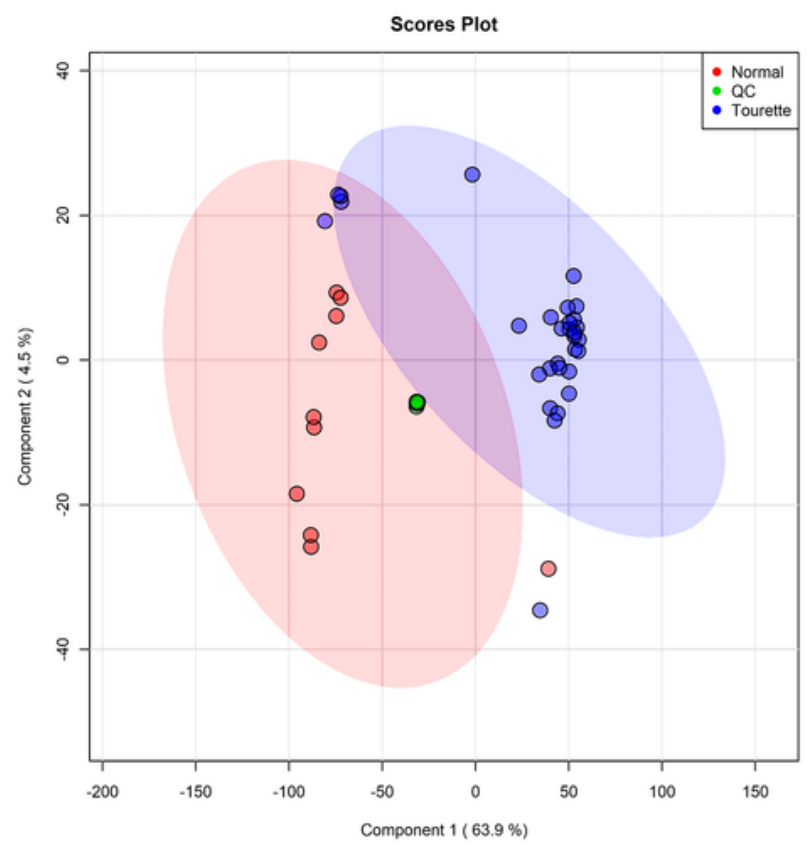

B
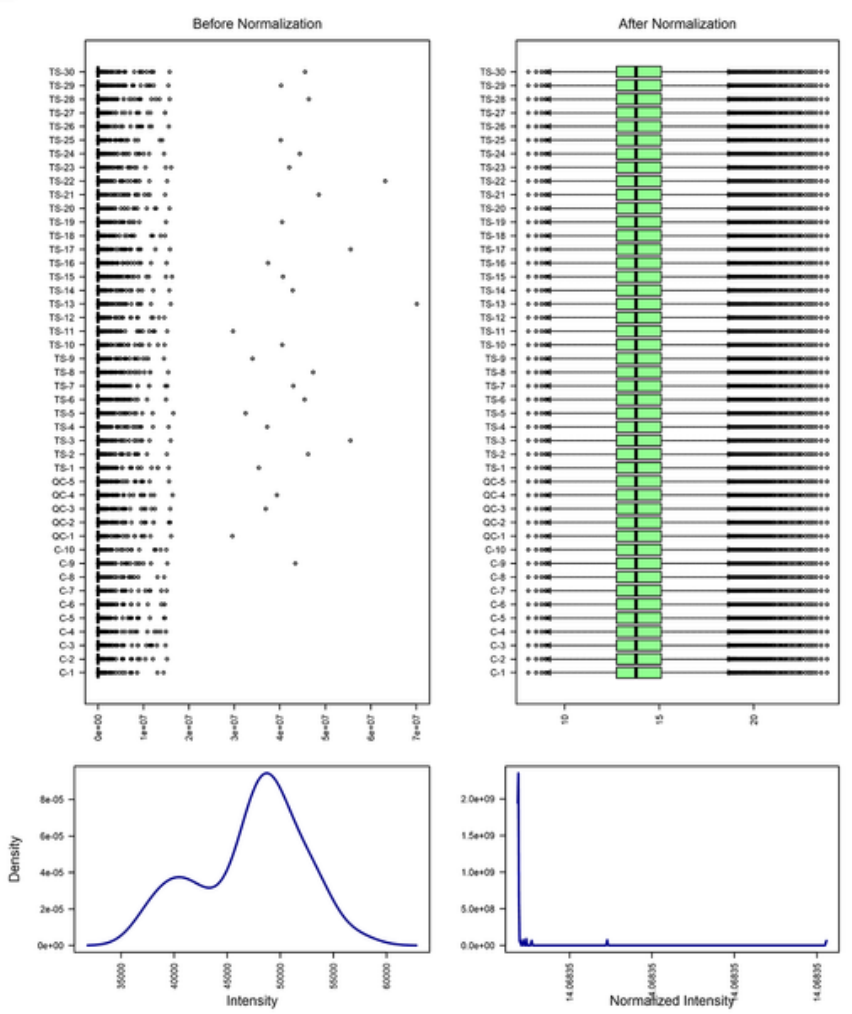

D

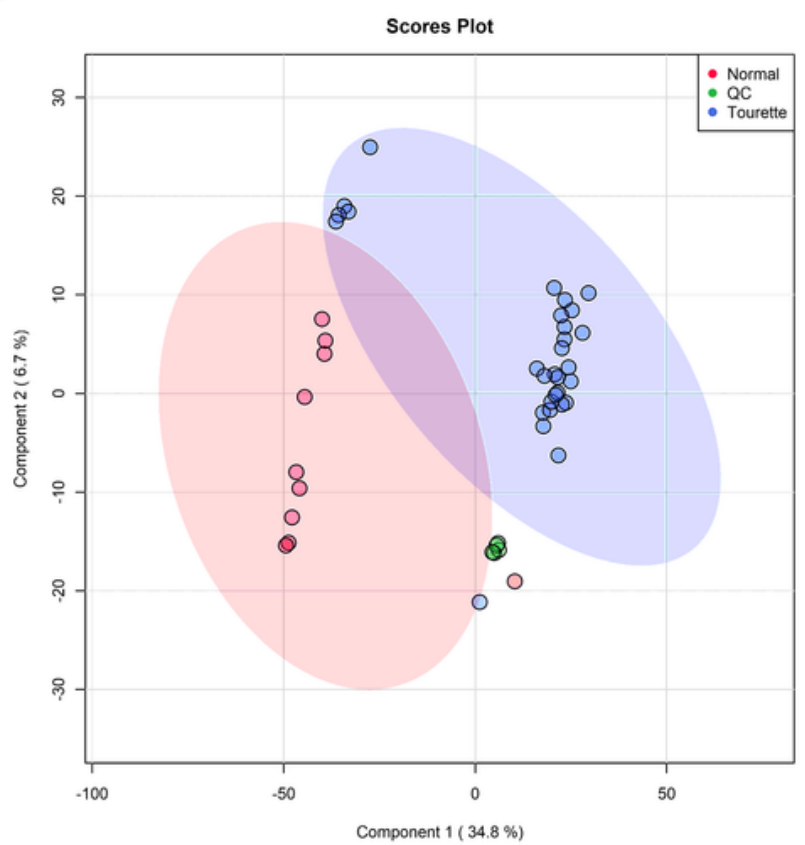

\section{Figure 1}

The sample distribution of TS patients and healthy controls. The distribution and intensity of input data values before (left) and after (right) normalization in positive ion mode (A) and negative ion mode (B) PLS-DA of plasma samples of TS patients vs. healthy controls in positive ion mode (C) and negative ion mode (D). The light red oval represents the $95 \% \mathrm{Cl}$ of the score calculated from each TS patients, and light purple oval represents the $95 \% \mathrm{Cl}$ of the score calculated from each healthy control. 
A $\quad$ B
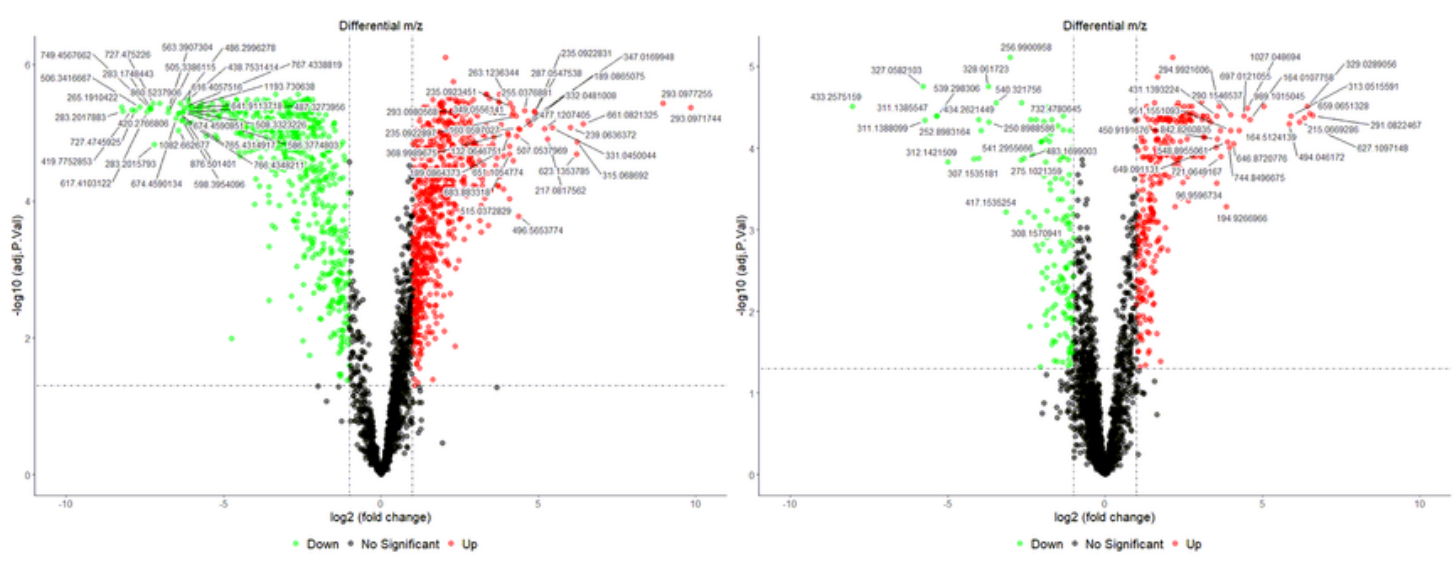

C

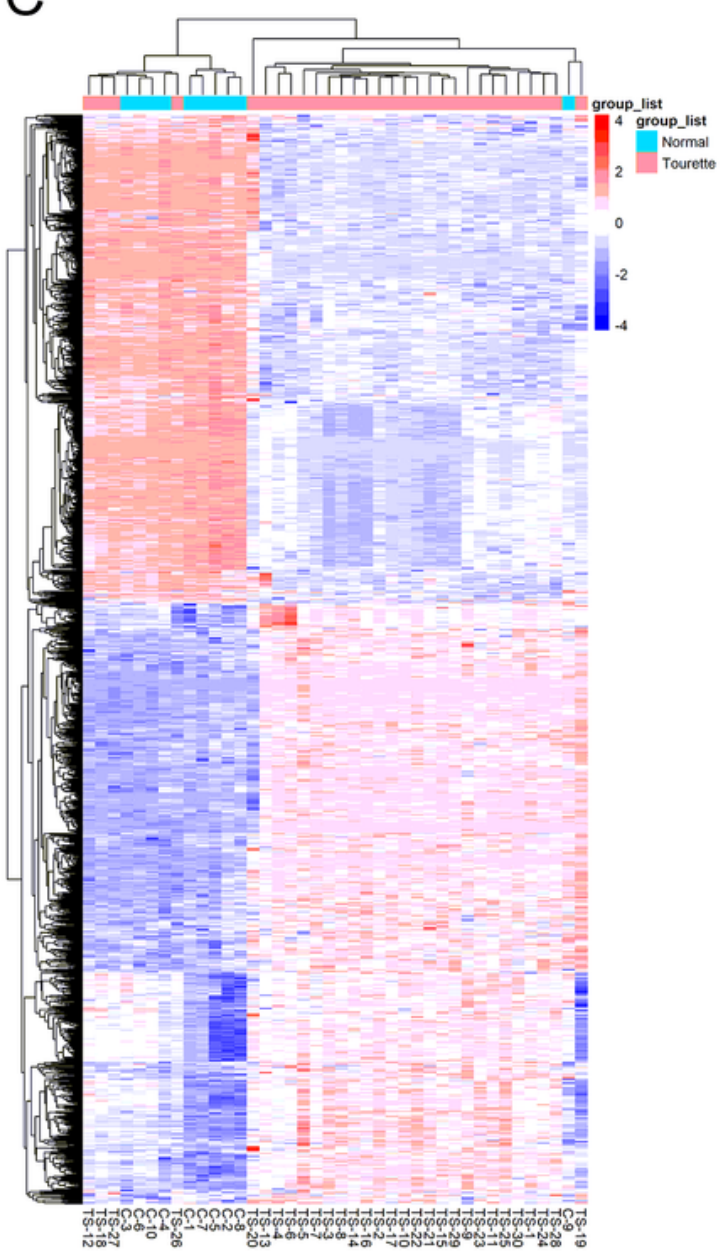

D

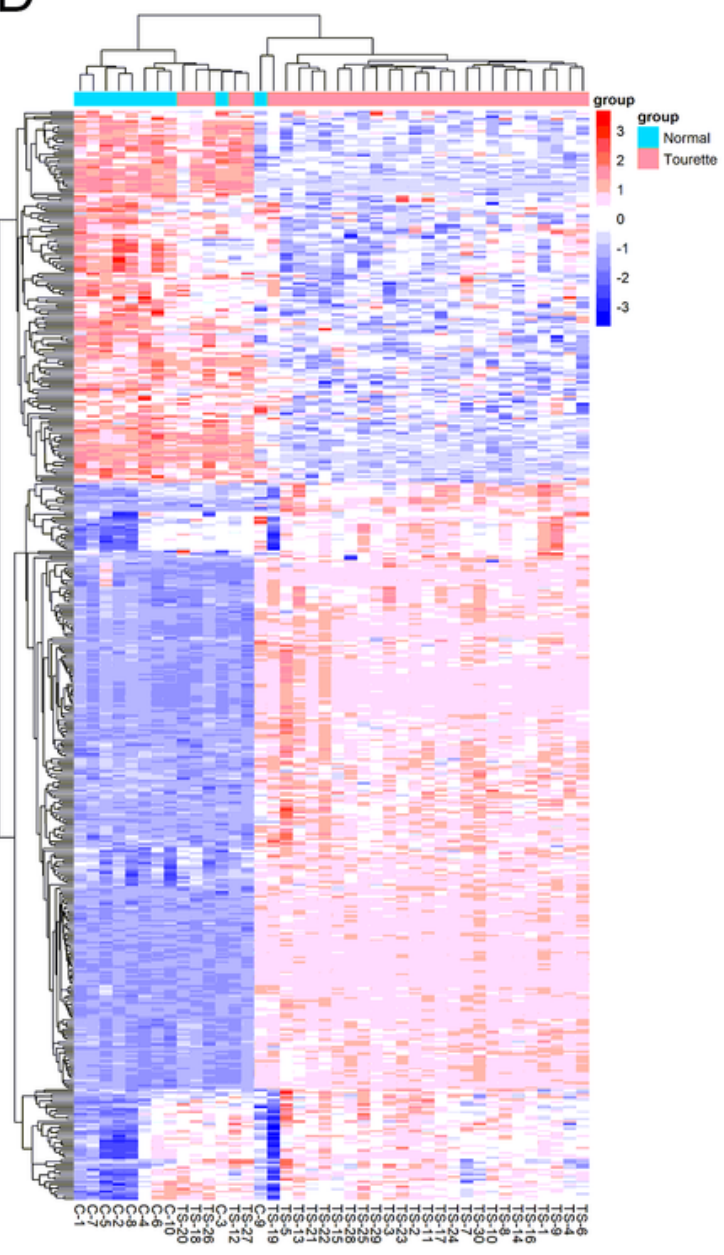

\section{Figure 2}

Different metabolites analysis for the data obtained from UHPLC-Q-TOF/MS non-target set. Volcano plot of different metabolites in positive ion mode (A) and negative ion mode (B). The volcano plot is a combination of fold change and t-test. The $x$-axis is $\log 2$ (FC), Y-axis is -log10 (adj.P.val). The red dots are $\log \mathrm{FC}>1$. The green dots are $\log \mathrm{FC}<-1$. Heatmap of clustering analysis of significant different 
compounds between TS patients and healthy controls in positive ion mode (C) and negative ion mode (D).

A

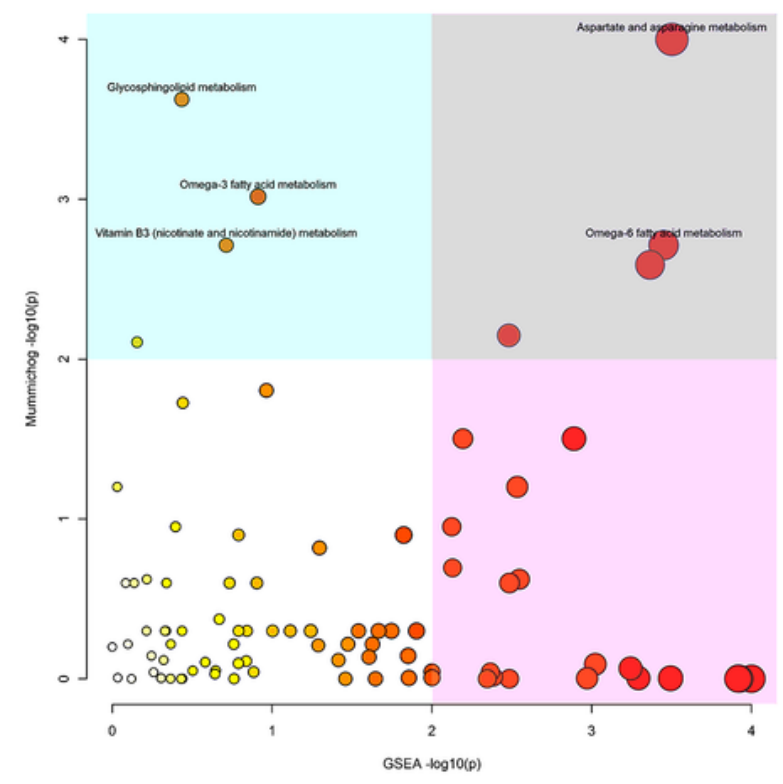

C

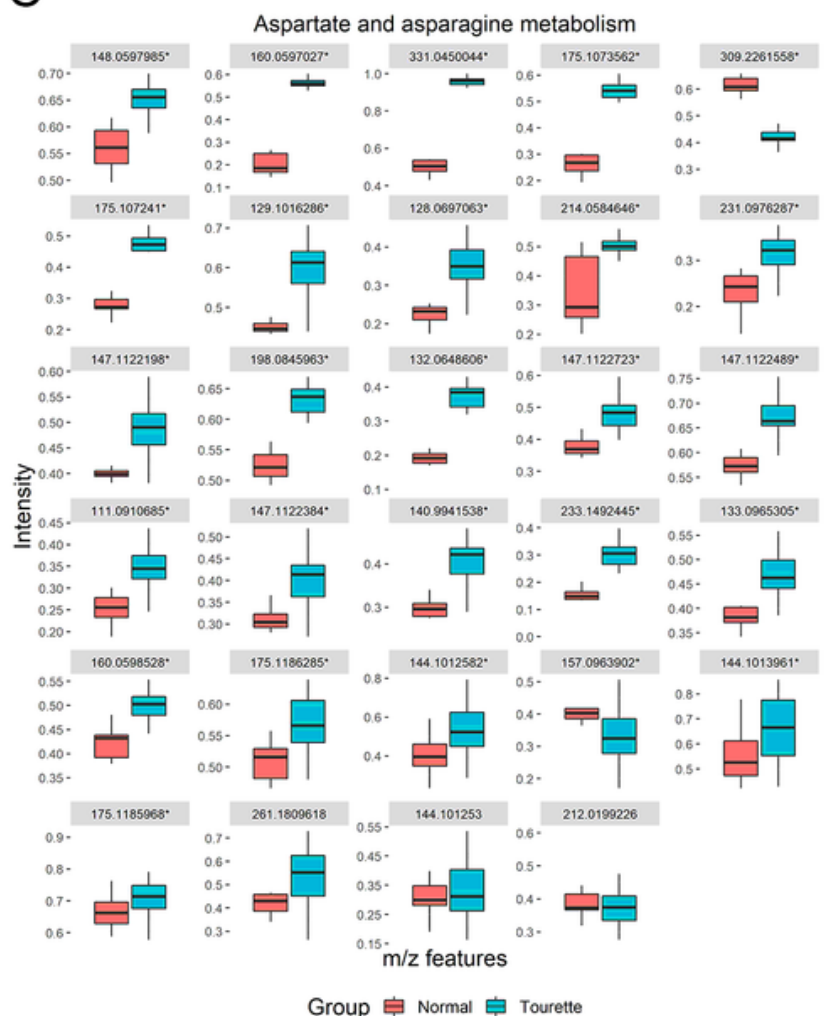

B

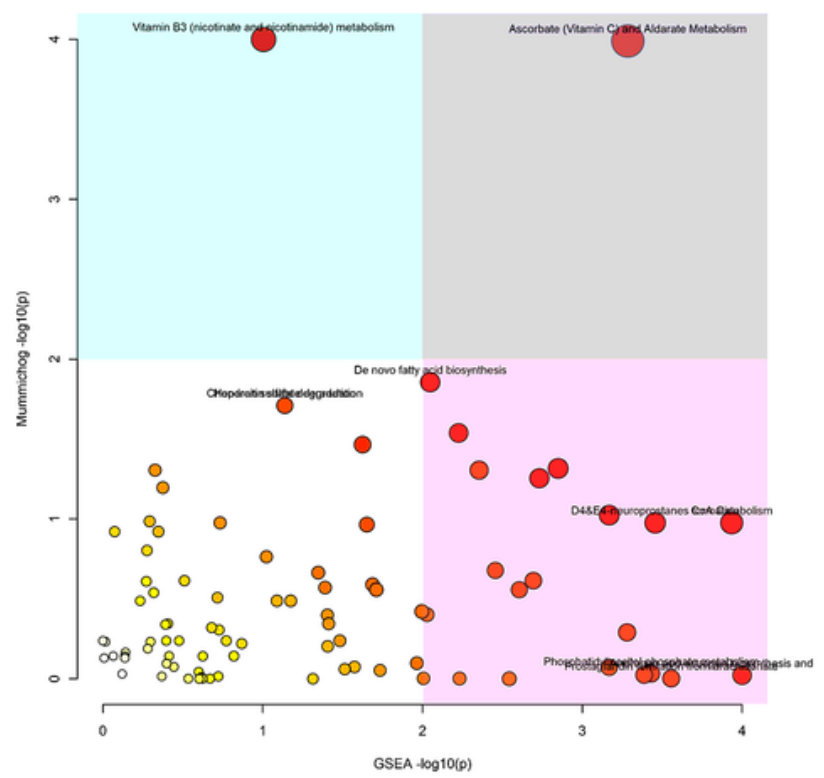

D

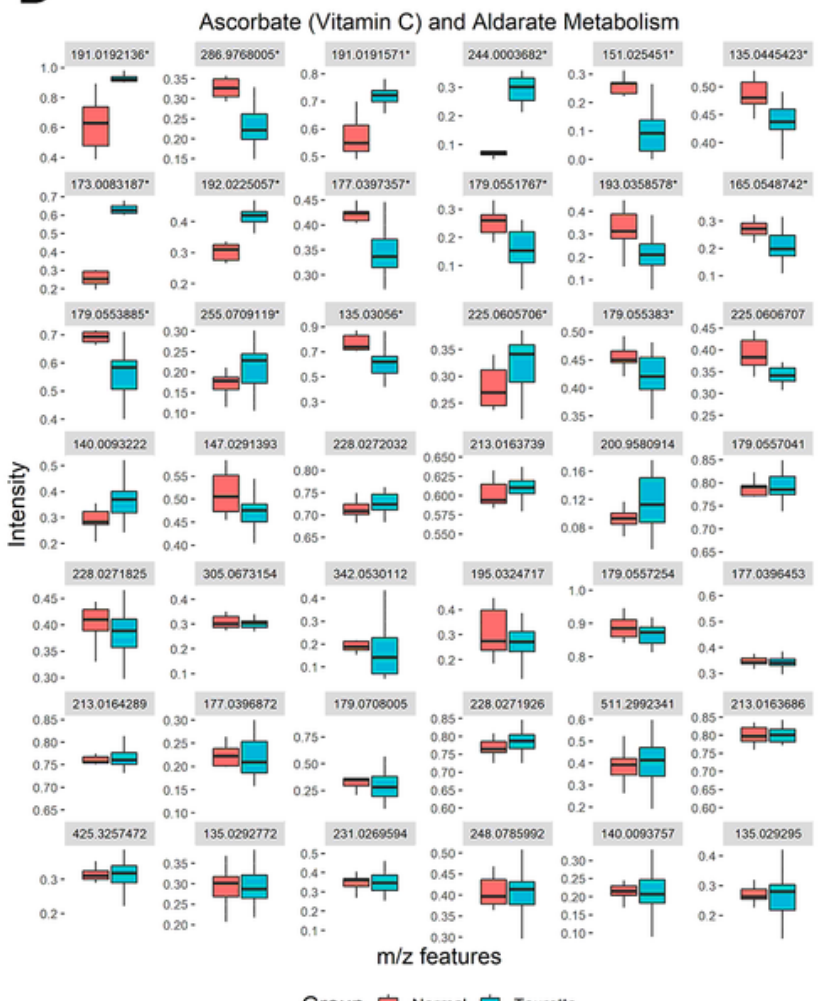

Figure 3

The enriched pathway analysis among significant different expression metabolites. The metabolism pathway enrichments of differential metabolites in positive ion mode $(A)$ and negative ion mode(B). The $\mathrm{x}$-axis is GSEA enrichment $-\log 10(\mathrm{p}), \mathrm{Y}$-axis is Mummichog enrichment $-\log 10(\mathrm{p})$. The matched 
metabolites from the different analyses in the Aspartate and asparagine metabolism pathway(C) and Ascorbate (Vitamin C) and alternate metabolism pathway(D).
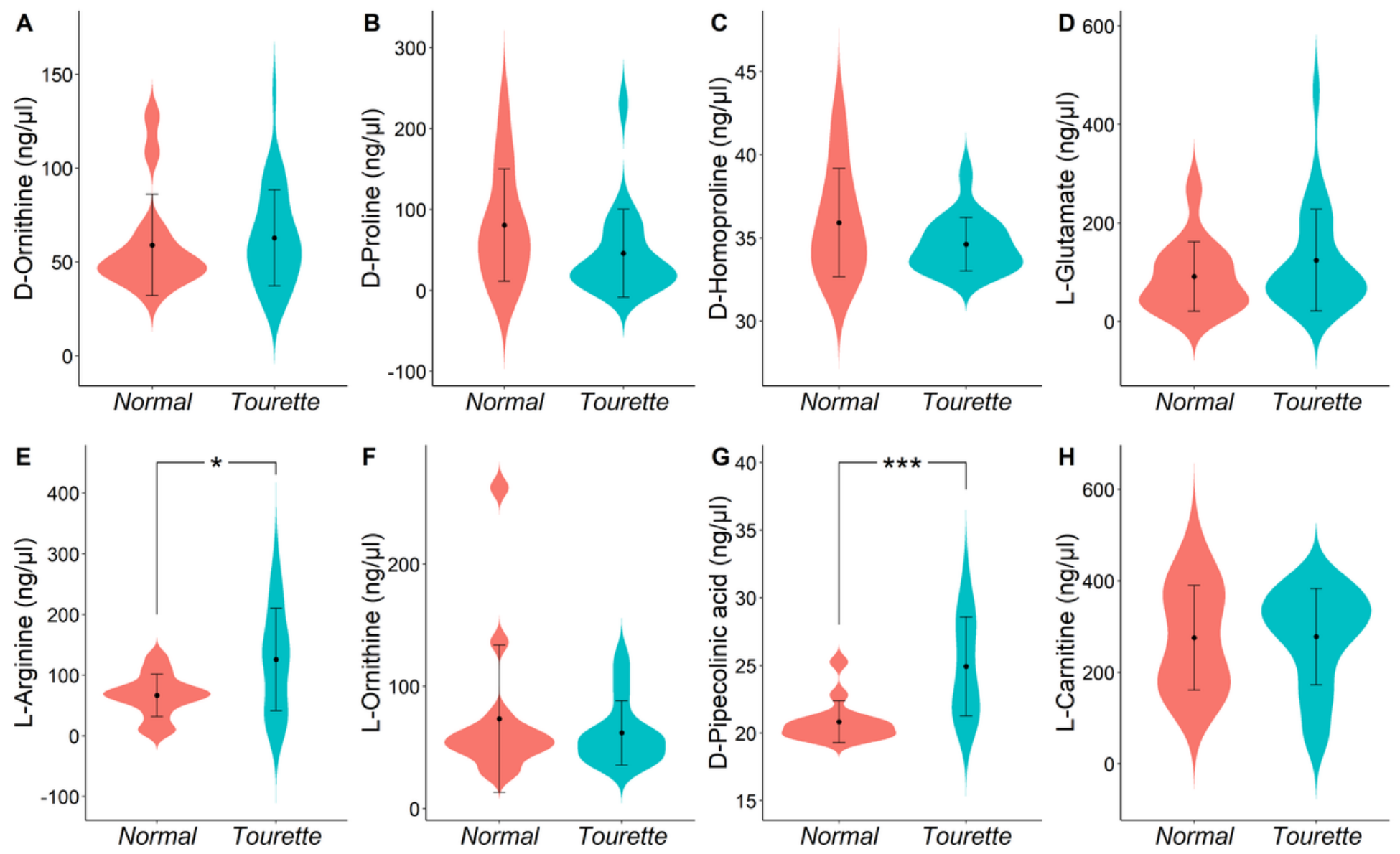

Figure 4

Eight candidate metabolites identified by target UHPLC/MS/MS quantitative analysis. D-Ornithine (A), DProlile (B), D-Homoproline (C), L-Glutamate (D), L-Arginine (E), L-Ornithine (F), D-Pipecolinic (G), LCarnitine $(H)$ levels between TS patients and healthy controls in plasma. The lines show interquartile ranges, dots show medians. ${ }^{*} \mathrm{P}<0.05,{ }^{*} * \mathrm{P}<0.001$. 
A
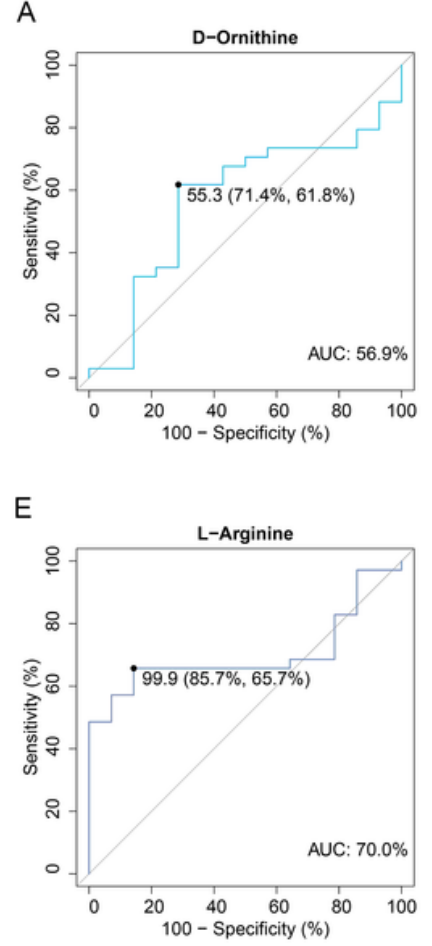

B

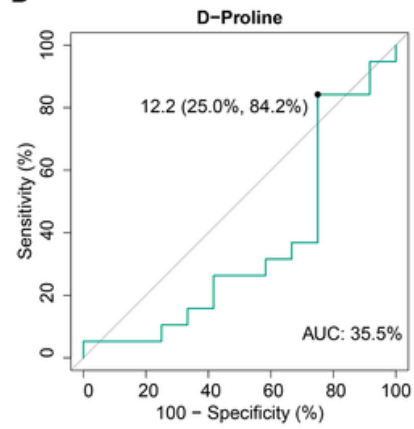

$\mathrm{F}$

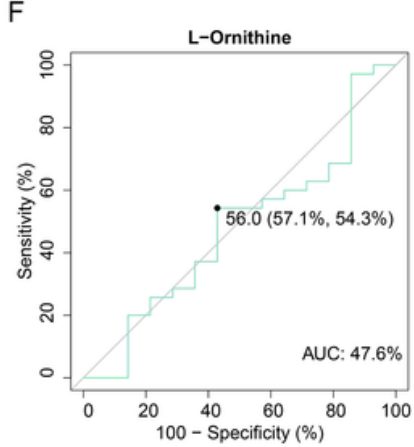

C

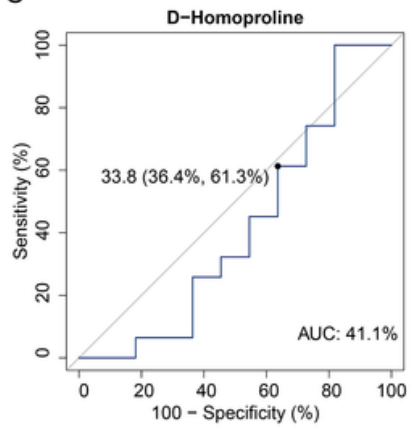

G

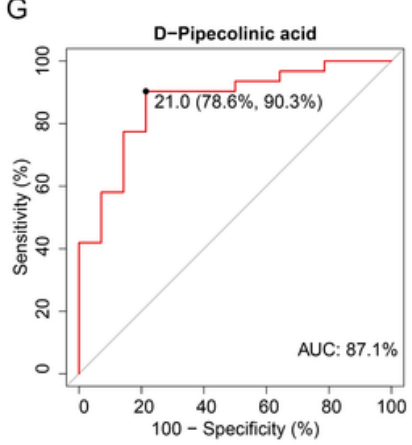

D

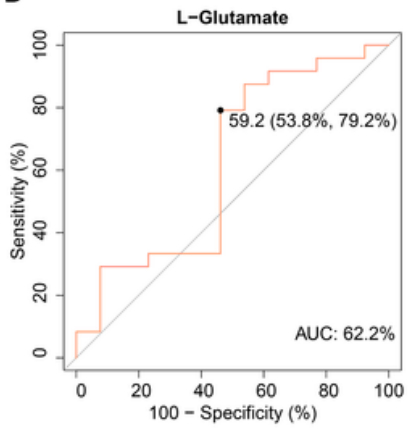

$\mathrm{H}$

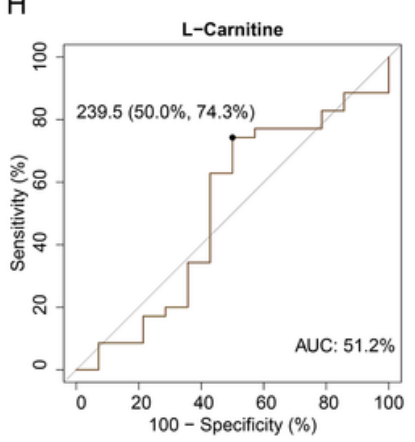

Figure 5

The ROC curve analysis of candidate metabolites amino acids in UHPLC/MS/MS targeted group. DOrnithine, AUC $=56.9 \%$, Youden index $=55.3(71.4 \%, 61.8 \%)(A)$, D-Prolile, AUC $=35.5 \%$, Youden index $=$ 12.2(25.0\%, 84.2\%) (B), D-Homoproline, AUC $=41.1 \%$, Youden index $=33.8(36.4 \%, 61.3 \%)(C)$, LGlutamate, $A U C=62.2 \%$, Youden index $=59.2(53.8 \%, 79.2 \%)(D)$, L-Arginine, $A U C=70.0 \%$, Youden index $=$ 99.9(85.7\%, 65.7\%) (E), L-Ornithine, AUC = 47.6\%, Youden index $=56.0(57.1 \%, 54.3 \%)(F), D-P i p e c o l i n i c$, AUC $=87.1 \%$, Youden index $=21.0(78.6 \%, 90.3 \%)(\mathrm{G}), \mathrm{L}-$ Carnitine, $\mathrm{AUC}=51.2 \%$, Youden index $=$ $239.5(50.0 \%, 74.3 \%)(H)$. AUC (50\%-70\%), low accuracy; AUC (70\%-90\%), moderate accuracy; AUC $(>90 \%)$, high accuracy.

\section{Supplementary Files}

This is a list of supplementary files associated with this preprint. Click to download.

- SupplementaryFigureS1.tif

- SupplementaryFigureS2.tif

- SupplementaryFigureS3.tif 